\title{
A multi-interface platform system for assistance and evaluation of disabled people
}

\author{
Chadi Fouad Riman ${ }^{\mathrm{a}, *}$, Hector Peralta $^{\mathrm{b}}$, Eric Monacelli ${ }^{\mathrm{b}}$, Yasser Alayli ${ }^{\mathrm{b}}$, Ali El Hajj ${ }^{\mathrm{c}}$ \\ and Imad Mougharbel ${ }^{\mathrm{d}}$ \\ ${ }^{a}$ Fahad Bin Sultan University, Tabuk, Saudi Arabia \\ ${ }^{\mathrm{b}}$ Versailles University, Versailles, France \\ ${ }^{\mathrm{c}}$ American University of Beirut, Beirut, Lebanon \\ ${ }^{\mathrm{d}}$ Lebanese University, Beirut, Lebanon
}

\begin{abstract}
In this work, an evaluation and assistance platform for people with disabilities is presented. This platform allows the control of different systems (PC games, robot, electric wheelchair) using different interfaces (mouse, joystick, etc.). The software facilitates the saving of experimental data for analysis, it can be automatically reconfigured to match user's ability, and it is operated locally or remotely through the internet. Quantitative indicators are defined for evaluation purposes and to determine the needed assistance and adaptation. The system is tested and results are presented and discussed.
\end{abstract}

\section{Introduction}

The treatment of people with disabilities is benefiting nowadays from advancement in technology [1]. This treatment may include an evaluation phase for observing and determining the capabilities and disabilities of the patient, a reeducation phase for reducing the disability by means such as practical exercises, and a compensation phase for overcoming the disability by an alternative system or method.

It is sometimes difficult for people with severe motor disabilities to use computer devices such as mouse, joystick, trackball, and other peripherals. In such cases (Fig. 1), the patient applies a force on the peripheral device which acts on the technical mobility aid (electrical wheelchair, walker, etc.), and the therapist does the necessary adjustment on the peripheral device and on the mobility aid (such as speed and response time)

*Corresponding author: Chadi Fouad Riman, PhD, Computer Engineering Department, Fahad Bin Sultan University, Tabuk, KSA. E-mail: chadi_riman@yahoo.com. by functional evaluation and analysis of the physical activity (kinesiology analysis). Such evaluation uses methods and tools which are mainly centered on the user's satisfaction, on the contribution of a technical assistance, and on comfort or pleasure.

Many computer-based evaluation systems for patients with motor disabilities are available. These systems suffer in most cases from some limitation. For example, some systems are specialized in one type of task, exercise, device, or evaluation. They allow very limited or no change in configuration, their evaluation is based on qualitative indicators which are, in many cases, subjective and inaccurate, and they lack advanced features such as guiding assistance and remote supervision [2]. A brief overview of many existing systems is given in Section 2.

In this work, an evaluation and assistance platform for people with disabilities is presented: "Platform for Interface and Assistance Evaluation" (PLEIA as French title acronym). PLEIA was mainly developed for several types of motor disabilities, including people with cerebral palsy or MCD (Motor Cerebral Deficiency), post stroke patients, quadriplegic patients, and 


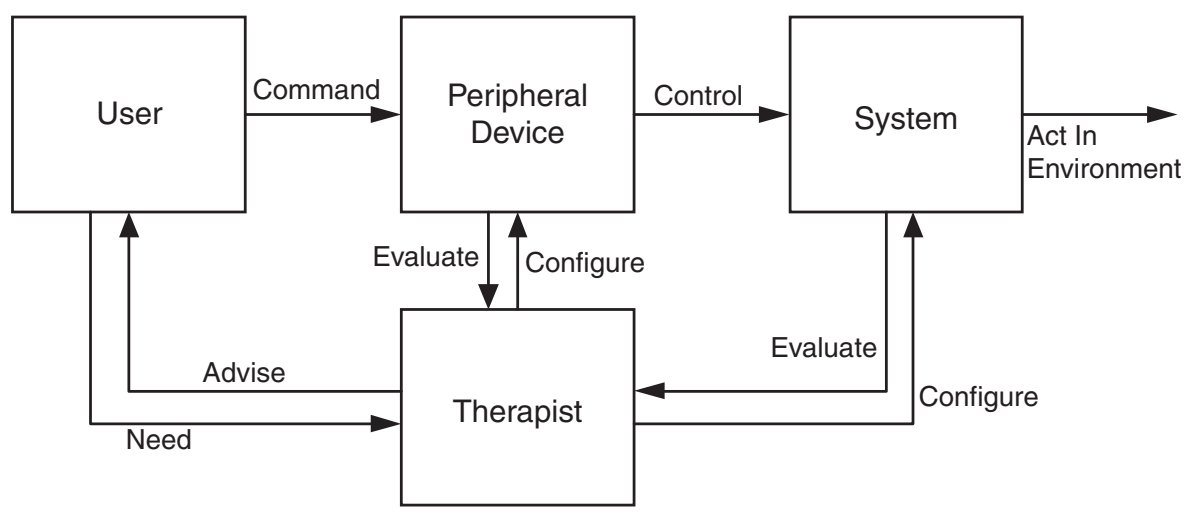

Fig. 1. Strategy for a normal therapy session.

persons who cannot control their movements because of weakness or spasm (myopathy, athestosy). Patients use PLEIA via various kinds of input-devices such as mouse, haptic joystick with force feedback, trackball, head tracker or others. This allows them to control computer-simulated systems (PC games), real objects (robots and toys), and other machines (electric wheel chair or walker). PLEIA can also be used by the occupational therapist and the medical team. For this purpose, quantitative indicators were defined and used for evaluation, assistance and adaptation. These indicators can also compare users' performance. The software facilitates the saving of experimental data in a database for analysis and sharing. It can be automatically reconfigured to match the user's ability, and can be operated locally or remotely through internet or intranet connections [3, 4]. PLEIA can also be used to generate configurations and/or test scenarios for different devices.

PLEIA, as compared to other systems in the same area [5-10], has the following integrated features:

1. PLEIA integrates a set of tools into a single platform, while most other systems are focused on one or few tools or tasks.

2. PLEIA has an assistance mode which helps the patient in reaching a goal. This feature is only found in [7].

3. PLEIA has an adaptation mode which automatically reconfigures the system according to the patient's ability. This feature is only found in [11]. Furthermore, after detecting the patient's moves, PLEIA can convert them to actions that comply with the intentions of the former.
4. PLEIA has a supervision service which allows the therapist to control and follow up a session locally or remotely. A limited version of this feature was found in [9].

This paper aims to describe and authenticate our work to date. Section 2 describes existing systems, whereas Section 3 introduces PLEIA, Section 4 defines its quantitative indicators, and, finally, its services are presented in Section 5.

\section{Existing systems}

Nowadays, there are many available systems whose purpose is to treat and help people with disabilities. Most of these systems, however, suffer from certain limitations (as has been indicated in the introduction). These systems can be classified into three categories: Adapted Interfaces, Rehabilitation Software Systems, and Robotic Assistance systems. Examples of these systems are:

\subsection{Adapted interfaces}

In this category, computer interfaces were developed to help a patient use various devices. The work in [12] describes a handheld Palm computer which was developed as an alternative solution for people who are not able to use the keyboard and the mouse properly. The case of patients who are unable to use the joystick of an electric wheel chair is also considered in [13]. The joystick is replaced with a system which uses signals coming from the contracting muscles of the forehead to drive the wheelchair. In [14], an intelligent 
controller was developed to help the patient using an electric wheelchair by predicting the user's intention, and by driving the wheelchair to the estimated goal. It is suggested in [15], to control neural prosthetic systems based on cognitive signals which can be directly read from the nervous system. A supervisory system can use these signals to manage the interaction between the patient and an external device such as a robot arm. A head-operated computer mouse was developed in [16] by using two tilt sensors placed in the headset to determine the head position and to drive the displacement of the mouse. The "Smart wheelchairs" developed in [17] identify and avoid obstacles in their way by using sensors and voice control with navigation assistance. The software aid developed in [11] adapts headoperated computer interfaces to special user needs by automatically adjusting the interface sensitivity.

\subsection{Rehabilitation software systems}

This subsection describes some software systems which were developed for the patient's evaluation and assistance. The system presented in [7] evaluates the "point and click" ability of a user based on many criteria and provides assistance to reach a target through a haptic interface. Two other low cost telerehabilitation systems were introduced in [5]: The first uses a force feedback joystick with simple twodimensional (2D) games, whereas the second uses a force feedback driving wheel with three-dimensional (3D) driving exercises. Another telerehabilitation platform [6] is intended for patients with a stroke-induced disability. This platform uses several haptic devices (joystick, driving wheel, pointing devices, keyboard) and includes remote (Internet) accessibility and videoconferencing. In [8], a telerehabilitation system for arm and hand therapy following stroke is presented. It consists of a Web-based library of status tests, therapy games, and progress charts, and can be used with a variety of input devices, including force feedback joysticks. In [9], Technical and Patient Performance Using a Virtual Reality-Integrated Telerehabilitation System is described. The system consists of the Rutgers Ankle prototype robot, a local PC and a remote PC over the Internet. In [10], exercises were built and used on a laptop with a force feedback joystick and a steering wheel for measuring motor dysfunction in Parkinson's disease. The exercises consist of tracking a continuously moving target (pursuit tracking), or moving to a predetermined target (step tracking). A haptic interface for hand evaluation and rehabilitation was developed in [18]. This system uses the DataGlove Rutgers Master II (RMII). Another system called "Rutgers Ankle" was also developed for lower extremity rehabilitation [18]. The built-in software provides exercises for different cases: Rubber ball exercise for strengthening the hand of a patient, and Virtual RMII exercise for the rehabilitation of post-stroke patients, and Virtual airplane exercise for lower extremity rehabilitation. Virtual reality by means of a head-mounted display was used in [19] for the purpose of improving patient walking skills through training with computer-generated obstacles. In [20], a low-cost telerehabilitation system for upper-limb dysfunction was presented. An example of a virtual driving environment was also shown using a commercial force feedback driving wheel. In [21], an exercise system combining arm ergometry with video gaming, called the GAME/sup Cycle, was developed. A haptic device with two active degrees of freedom and a tendon-driven transmission system was developed in [22].

\subsection{Robotic assistance systems}

This category is concerned with robotic assistance to disabled people. As an example, a manipulator arm (ARPH project) mounted on a mobile robot was developed in [23] in order to assist a person in moving and controlling an object. Another example consists of a passive walking support system called "RT Walker" [24] which uses environment information to help the user in avoiding obstacles. It is equipped with sensors and actuators for discovering and locating obstacles, for sensing the direction of the motion, and for controlling the brakes. This system does not have a motor, which makes it safer during users' walk.

\section{PLEIA description}

PLEIA is a platform which has two main purposes: evaluate a person's dexterity to use a computer and manipulate a mobility aid such as an electric wheel chair. Different tests are used to achieve these goals. Patients' tests can be applied using different interfaces such as joystick (with or without force feedback), mouse, trackball, and head tracker. These interfaces are used to control two-dimensional (2D) computersimulated exercises, three-dimensional (3D) objects (robots, toys), and mobility systems (electric wheel 
chair, walker). The software facilitates the saving of a patient's exercise or of test data in a database for analysis and sharing. It can be automatically reconfigured to match the user's ability, and can also be used to generate configurations and/or test scenarios for different devices [25].

PLEIA can be accessed locally or remotely through internet or intranet connections [3, 4]. Many users can be simultaneously connected to the server where the experiment is being conducted. It is, therefore, possible to remotely execute a test, modify experiment parameters, chat between server and clients, exchange scenarios and experiment parameters, and help the user in achieving a goal by using another pointing device (joystick, mouse or others) in parallel.

PLEIA can also be used by the occupational therapist or by the medical team. For this purpose, quantitative indicators are defined (Section 4) and used for evaluation, for determining the needed assistance and adaptation, and for comparing user's performance. The upcoming subsections describe the PLEIA operation, PLEIA Functional Blocks, PLEIA Services, and the creation and configuration of test scenarios.

\subsection{PLEIA operation}

PLEIA is a platform (Fig. 2) which is added into the middle of the standard therapy session presented in the introduction (Fig. 1). PLEIA contains three modules: The Kernel which coordinates the activities of the other modules and controls the system, the Functional Blocks (Fig. 3) module which implements the type of exercise ("2D": on computer, "3D": on real object like toys, or "Functional": on assistive systems like powered wheelchair), and the services module which implements the services which are provided by PLEIA (Evaluation, Supervision, Assistance and Adaptation). In Fig. 2, the patient applies a force on the peripheral device, and its new position is used by the Functional Block module to calculate the necessary task achievement (such as moving computer cursor or electric wheel chair). The Kernel sends the calculation result to the services module and to the system (Electric wheelchair, Robot, Toy, computer screen). The services module may correct the result and passes it back to the Kernel which uses it to act on the system (computer cursor or wheel chair).

\subsection{PLEIA functional blocks}

PLEIA multi-platform (Fig. 3) defines 3 different platforms: PLEIA computer software exercises (2D), PLEIA object manipulation exercises (3D), and PLEIA functional application.

PLEIA 2D module supports the control of twodimensional computer-simulated exercises. Four types

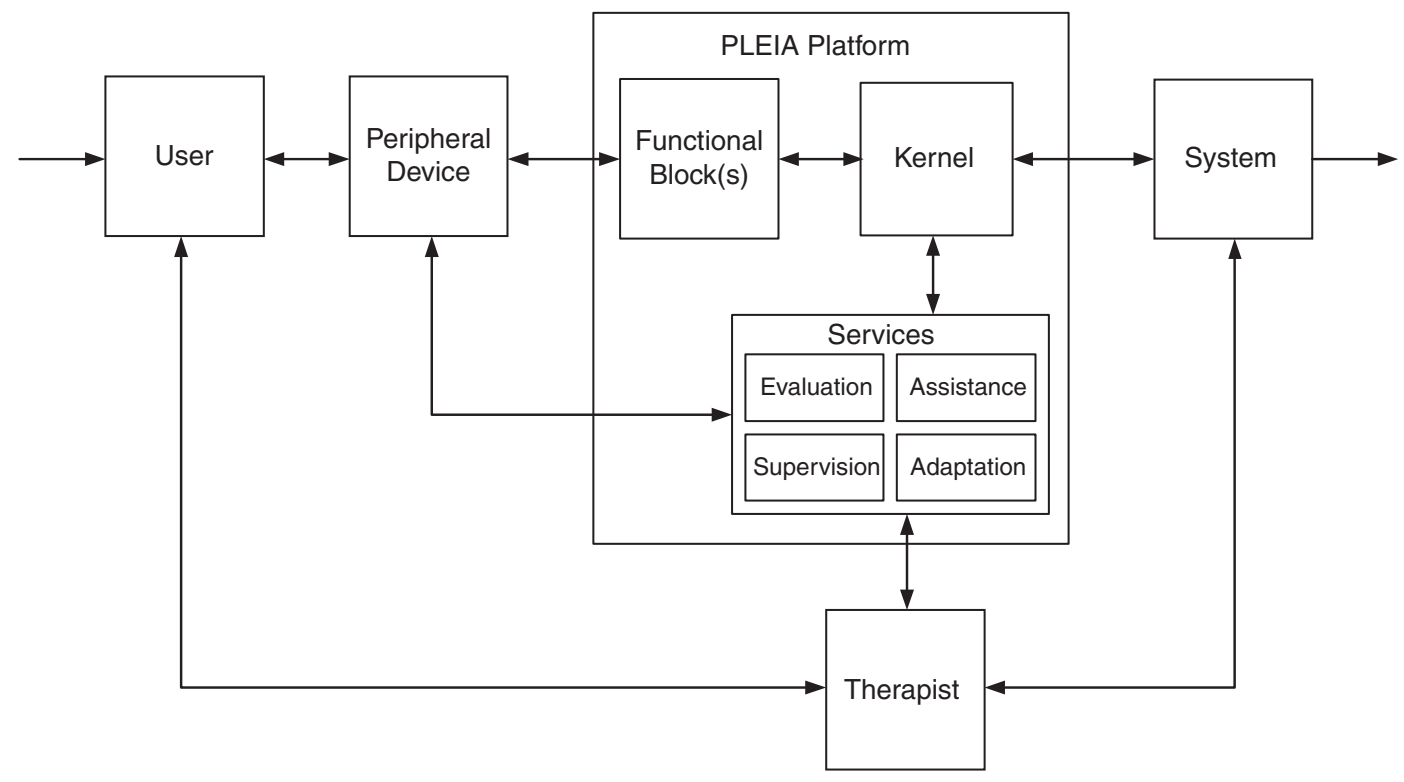

Fig. 2. PLEIA strategy. 


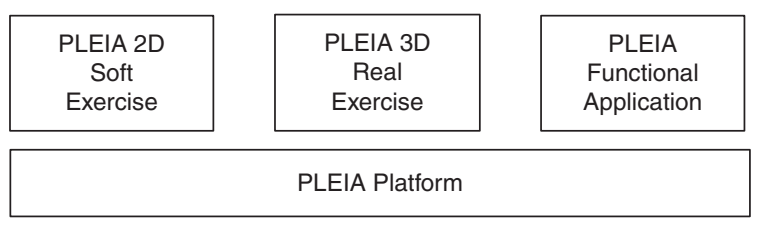

Fig. 3. PLEIA functional blocks.

of exercises are proposed by an occupational therapist team: The "Reach Targets" exercise (Fig. 4a) which requires reaching all targets in a specified order, showing the next target in green and the others in red. The "Click Targets" exercise (Fig. 4a) is similar to "Reach Targets" exercise but which requires, in addition, clicking at each reached target. The "Follow Path" exercise (Fig. 4b) requires reaching all the targets while following a drawn path. In the "Drag and Drop" exercise (Fig. 4c), it is required to reach a target, pick it up, move it and drop it in the next target. This exercise is repeated for all the targets. These computer exer- cises are in two modes of operation: PLEIA Abstract and PLEIA Tuned. In PLEIA Abstract mode, the user manipulates simple objects (circles, rectangles) on the computer screen. This mode is appropriate for most types of evaluations. In PLEIA Tuned mode, the user can select images that fill screen objects, or choose a special background and a moving cursor shape (Fig. 5). This mode is preferred by children according to therapist's recommendations.

PLEIA 3D module deals with two real objects: Robots and Toys. In the first case, a small "Khepera" robot [28], which is widely used for education and research purposes, is moved in all directions with different speeds. If the robot reaches an obstacle, it reverses its direction for one second and notifies the user's haptic interface (joystick) with reverse force feedback (Fig. 6).

PLEIA Functional Application module supports the control of mobility aid systems such as an Electric Wheel Chair or an Electric Walker. This mode can be (a) Reach/click targets

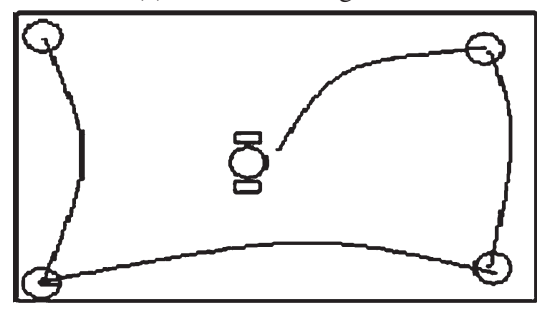

(b) Follow path

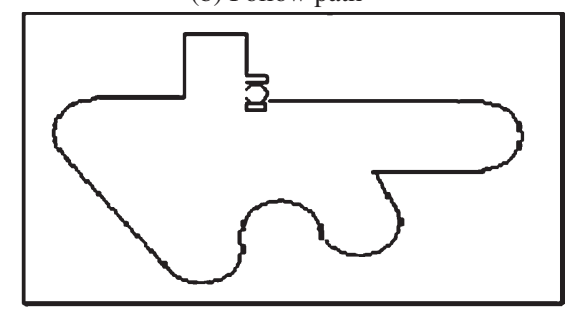

(c) Drag and drop

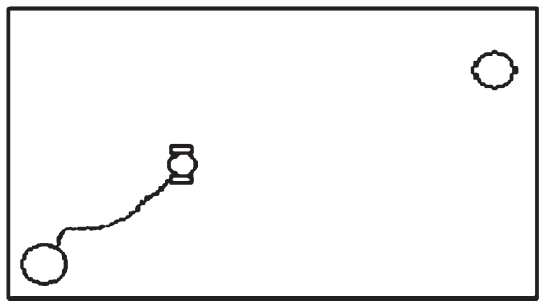

Fig. 4. PLEIA abstract.

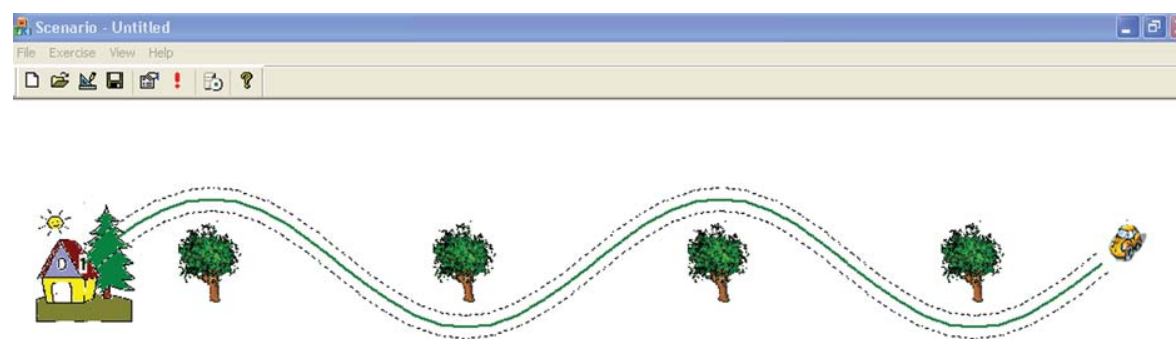

Fig. 5. PLEIA tuned. 


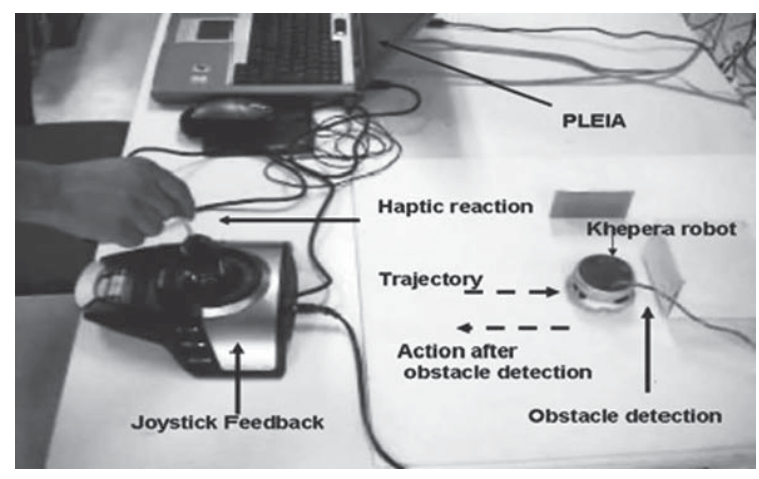

Fig. 6. Force feedback used for a haptic interface with Khepera Robot for educational and therapy sessions in PLEIA.

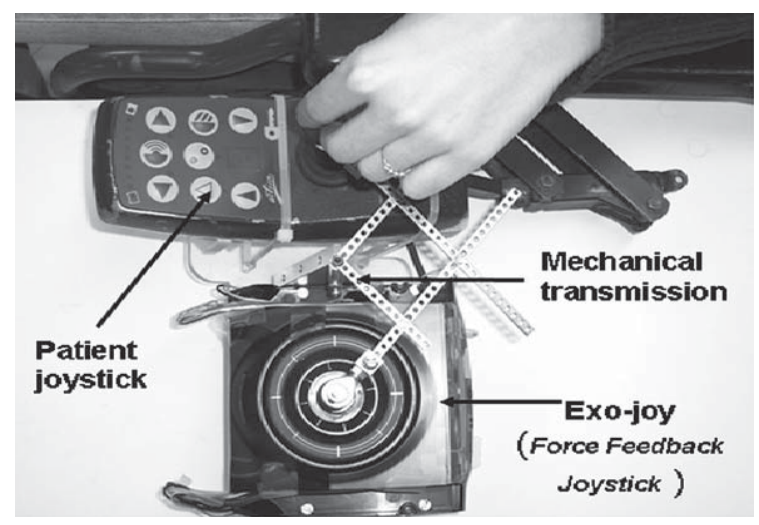

Fig. 7. Exo-joy structure used for the control of electric wheelchair joystick.

used for training purposes in which the user remotely manipulates a real electric wheel chair in a real environment. It can also be used to remotely assist a patient who is moving on an electric wheel chair. In the case of different types of wheelchairs, a device called exo-joy is built in order to translate the movements from a normal force feedback joystick to the built-in wheelchair joystick movement (Fig. 7). PLEIA uses a wireless remote control of exo-joy, which is located on the user's electric wheelchair's joystick.

\subsection{PLEIA services}

PLEIA offers 4 services: Evaluation service, supervision service, assistance service, and adaptation service.

The Evaluation service is used to provide an online or offline evaluation of a user. The data used for evaluation is collected online and analyzed. The frequency of data collection is $10 \mathrm{~Hz}$. An online feedback about an exercise performance can also be sent to the user in visual form, audio form, or through vibrations.

The Supervision service is used by the therapist in order to supervise a session. The supervision can be online during the test or offline by examining and analyzing test results after the test. It can be local on the same computer and location, or remote at another computer or location. PLEIA can handle two input devices at the same time (such as two joysticks or one joystick and one mouse), on the same computer, or on two networked computers. This feature allows the patient and the supervisor to cooperate in achieving a certain goal. For example, the patient can get force assistance from the supervisor joystick (human user help) to reach the targets. It is also possible to have a remote chat between patient and supervisor.

The Assistance service is used to provide assistance or training to the user during an exercise in order to reach a goal. For example, the user can get force assistance from the joystick to reach a target. This force can be an attraction force toward a target or a path, or a repulsion force to avoid an obstacle. It is implemented using a fuzzy logic inference (FIS) system, which is detailed in Section 5. This type of assistance is computer generated assistance which is different from the above mentioned human supervised assistance.

The Adaptation service is used to compensate a user's deficiency such as an inability to move the Joystick in one direction. This mode uses a fuzzy FIS controller in which the fuzzy logic rules are changed online during the exercise or offline after the exercise. The online mode was implemented using the reinforcement techniques presented in [26], and the offline mode was implemented using the Fuzzy C-mean technique explained in [27]. More details on this mode are given in Section 6. Assistance and Adaptation can only be achieved when using a pointing device with force feedback (joystick).

\subsection{Creation of a configuration and test scenarios}

A test scenario consists of different components used in a test (Fig. 8) such as targets, objects, obstacles and paths. Test scenarios can be generated, saved in text files for future use, and copied to other computers or therapists for sharing. A test scenario can also be modified and saved by a therapist. The therapist can also change the test configuration such as the speed, the feedback, distances from obstacles, the 


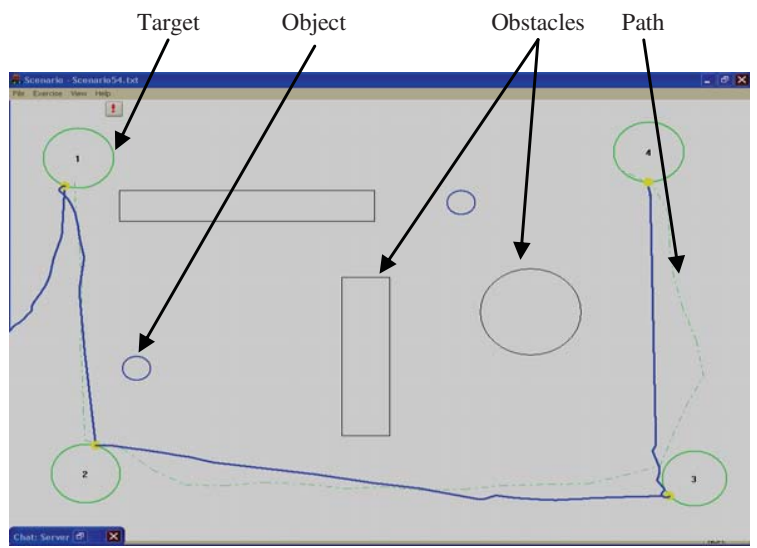

Fig. 8. PLEIA multi-platform software.

sound effects, the computer pointing device, and others. Such changes are important for proper therapy sessions.

\section{Quantitative evaluation indicators}

Important features of PLEIA are the quantitative indicators used for analyzing test results. Current therapists assumptions based on subjective analysis can be improved with measures derived from tests performed by the patients [29]. Using PLEIA, it is possible to quantitatively evaluate a patient's performance, compare two patients' performance, and record the evolution of a patient's performance.

PLEIA defines two types of indicators: task indicators which evaluate the direct test result, and comfort indicators which evaluate patient's behavior during the test.

Task indicators are first order indicators which give a direct classification of the user's performance during a test. Three task indicators are defined: time, distance, and collision/failure. The time indicator measures the total test accomplishment time. The distance indicator measures the total test distance. The collisions/failure indicator measures the total number of collisions with different obstacles during a test and indicates whether the user failed to accomplish the test or not.

Comfort indicators evaluate the user ability in manipulating the input device (joystick, mouse, trackball, etc.). Given the test trajectory $\left(x\left(t_{i}\right), y\left(t_{i}\right)\right)$, two comfort indicators are defined: Charge of command and Regularity of command. These indicators are still under study and other indicators are being proposed by the therapists for future PLEIA versions.

The charge of command indicator measures the variation of the direction of the effort applied by the user on the input device during a test. A small variation indicates little effort exercised by the user. The instantaneous charge of command indicator $v$ is first defined as the discrete derivative of the error angle (between actual and optimum movements over a partial distance) with respect to time:

$$
v(i)=\frac{\Delta(\theta)_{(i)}}{\Delta(t)_{(i)}}
$$

where $\theta$ is the angle between $\left(y_{i+1}, y_{i}\right)$ and $\left(x_{i+1}, x_{i}\right)$, and $\Delta \theta$ is the angle difference between current move (angle connecting $\left(y_{i+1}, y_{i}\right)$ and $\left.\left(x_{i+1}, x_{i}\right)\right)$ and the optimum move (angle connecting $\left(y_{\text {target }}, y_{\mathrm{i}}\right)$ and $\left.\left(x_{\text {target }}, x_{\mathrm{i}}\right)\right)$

The optimal movement is on the straight line linking the object to target.

The global charge of command is then defined as the mean of the instantaneous charge of command:

$$
\Gamma=\frac{\sum_{i=1}^{N}|v(i)|}{N}
$$

$N=$ points in the trajectory.

The regularity of command indicator measures the movement uniformity during a test. Whenever the movement's speed is nearly constant, the command is more regular. If the speed varies with time or the user stops often during the test, the command loses some regularity.

The total moved distance during a test at time $t_{i+1}$ is:

$$
d_{i+1}=d_{i}+\sqrt{\left(x\left(t_{i}\right)-x\left(t_{i+1}\right)\right)^{2}+\left(y\left(t_{i}\right)-y\left(t_{i+1}\right)\right)^{2}}
$$

where $d_{i}$ is the last total moved distance during a test at time $t_{i}$.

The speed (local regularity of command) is given by:

$$
w(i)=\frac{\Delta d_{i}}{\Delta t_{i}}
$$

The regularity indicator is calculated as the average speed variation over the trajectory:

$$
\Lambda=\frac{1}{m} \sum_{i \mid w(i) \neq 0}\left|\frac{\Delta w(i)}{\Delta t(i)}\right|
$$

where $m$ is the number of points with $w(i) \neq 0$. 


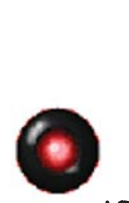

12

1

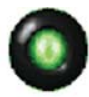

3

5

10

(8) 8

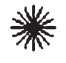

(e)

6

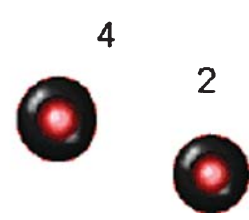

11

9

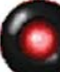

7

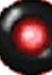

Fig. 9. Patient suggested test by therapist.

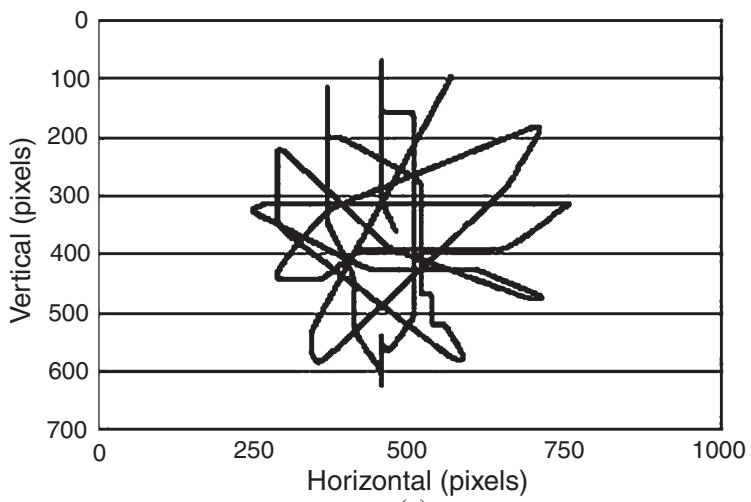

(a)

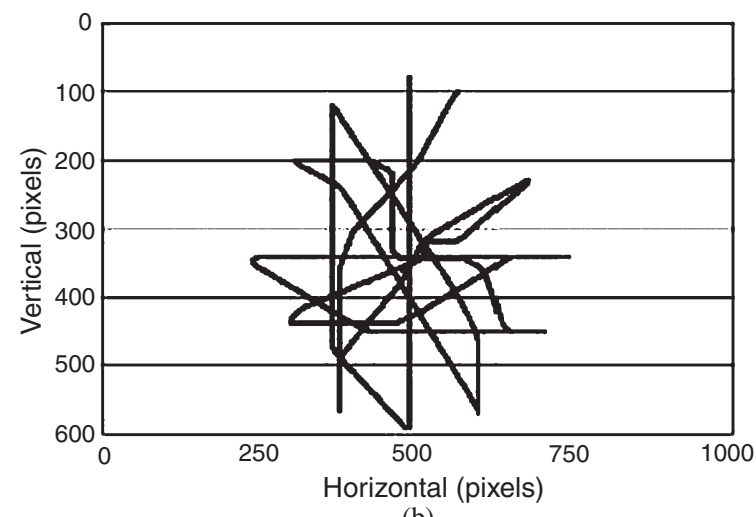

(b)

Fig. 10. Normal subject trajectories: (a) 1st try (b) Last try.
For any of the above indicators, an improvement indicator is used to describe the improvement of test's performance of a specific patient.

It is given by:

$$
\tau=\frac{\text { indicator } X(\text { test } 2)-\text { indicator } X(\text { test } 1)}{\text { indicator } X(\text { test } 1)}
$$

where indicator $X$ can be any of the mentioned indicators

For illustration purposes, a test was designed by a therapist to reach 12 goals in a specific order (Fig. 9). First, the test was performed by normal subject to get typical normal values (Fig. 10). Next, two set of tests (separated by 15 days) were made with a motor
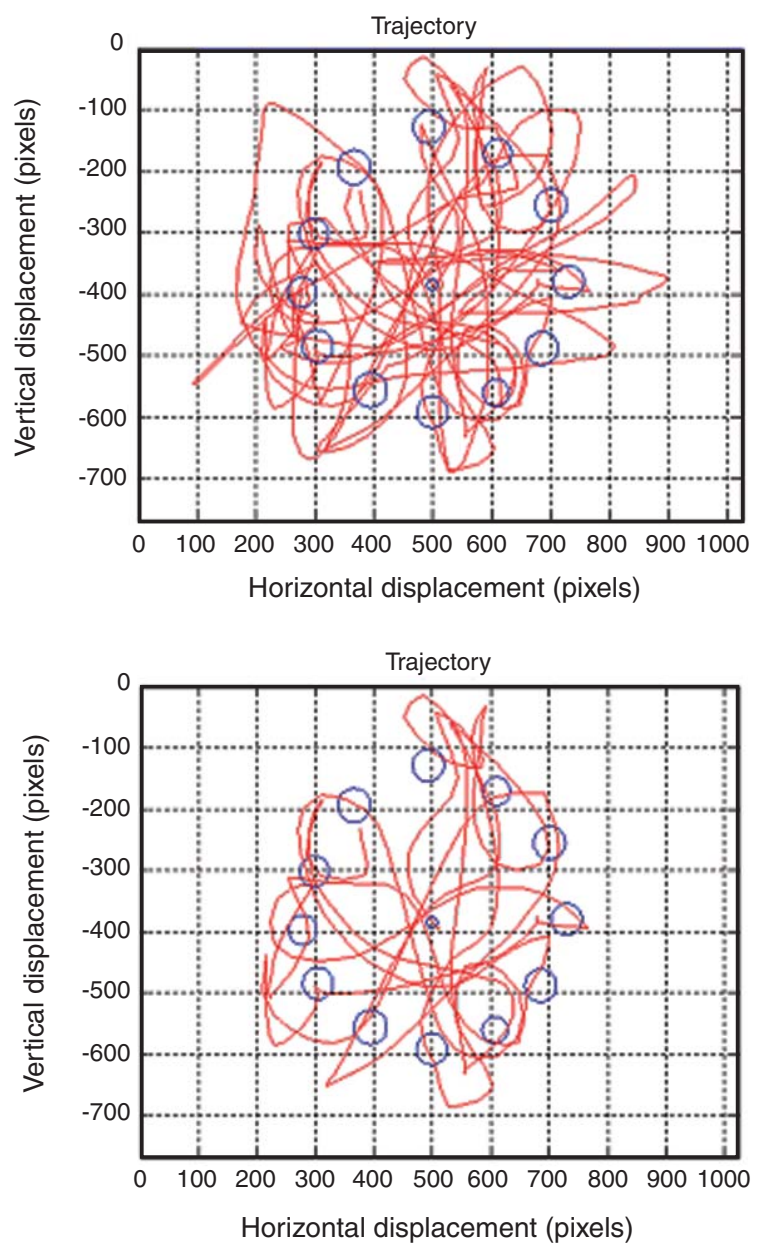

Fig. 11. Patient trajectories in the two tests. 

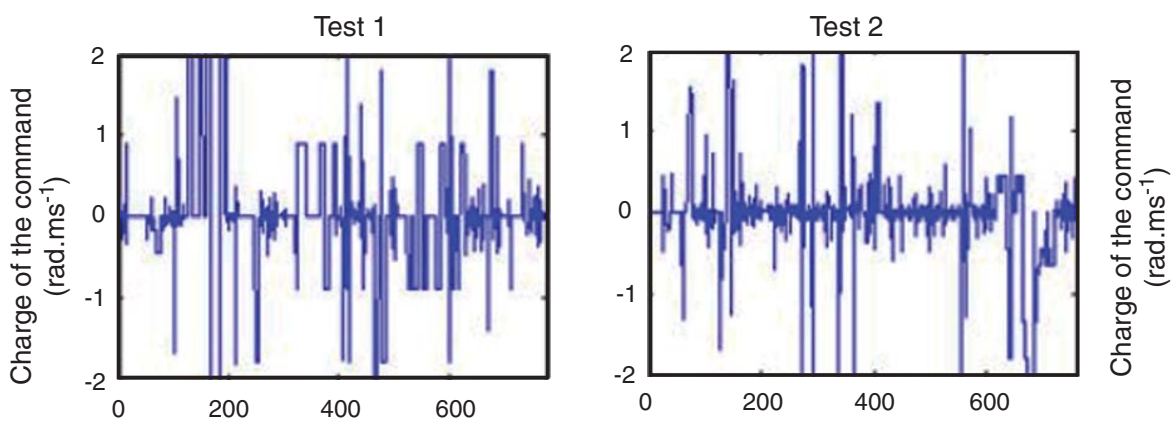

Fig. 12. Charge of command for the two tests.
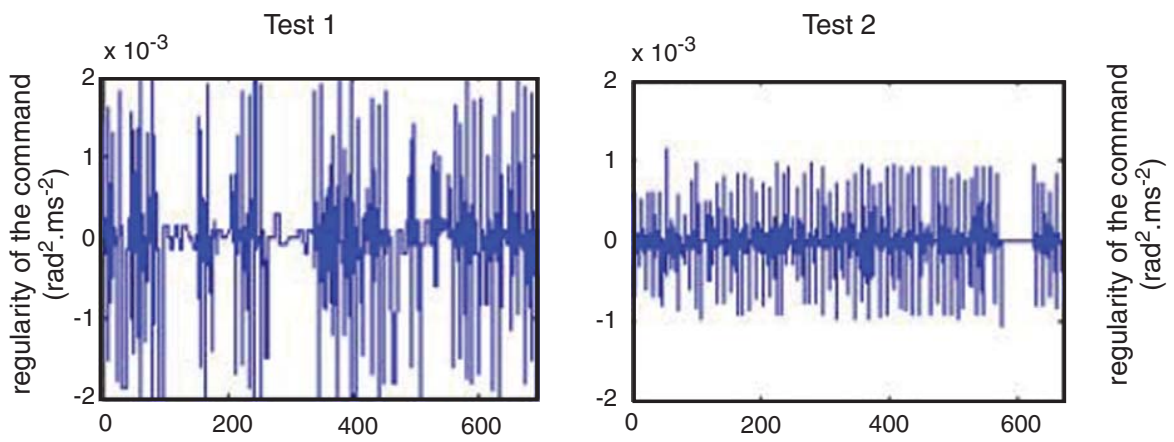

Fig. 13. Regularity of command for the two tests.

Table 1

Patient test indicators, compared to normal subject

\begin{tabular}{lccc}
\hline & Time (s) & Charge & Regularity \\
\hline Normal test 1 & 39 & 0.15 & 0.20 \\
Normal test 2 & 29 & 0.10 & 0.12 \\
Patient test 1 & 52.2 & 0.40 & 0.44 \\
Patient test 2 & 39.9 & 0.28 & 0.21 \\
Improvement & $-24 \%$ & $-31 \%$ & $-52 \%$ \\
\hline
\end{tabular}

handicapped patient (a 10-year-old quadriplegic girl) in order to quantify her learning improvement. Figures 11-13 show the trajectories made by this patient in the two tests respectively, the corresponding charge of command, and the corresponding regularity of command.

The protocol followed to perform the test was:

- Explain the general idea to the user.

- Explain the procedure to the user and show him what to do.

- Perform the test once in front of the user.
- Let the user try the experiment with our help twice without saving the results.

- Start the test and save the results. Help is only given orally to the user.

- Repeat each test 5 times for each user. The average results were taken into account in order to compensate for any learning curve or transient mistakes that might occur.

The tests indicators (Table 1 ) show a respective improvement of $24 \%, 31 \%$ and $52 \%$ in the time, charge and regularity of command indicators, which means that the patient has better control of the device, and that the movement is less perturbed (charge of command) and more continuous (regularity of command). This is due to the training done by the patient during the 15 days period on using the system.

We compared these results to a normal subject. This gives us an indicator of the optimal values. This example shows that the quantitative indicators can be a valuable tool for therapists to evaluate a patient and follow-up her/his learning progress. 


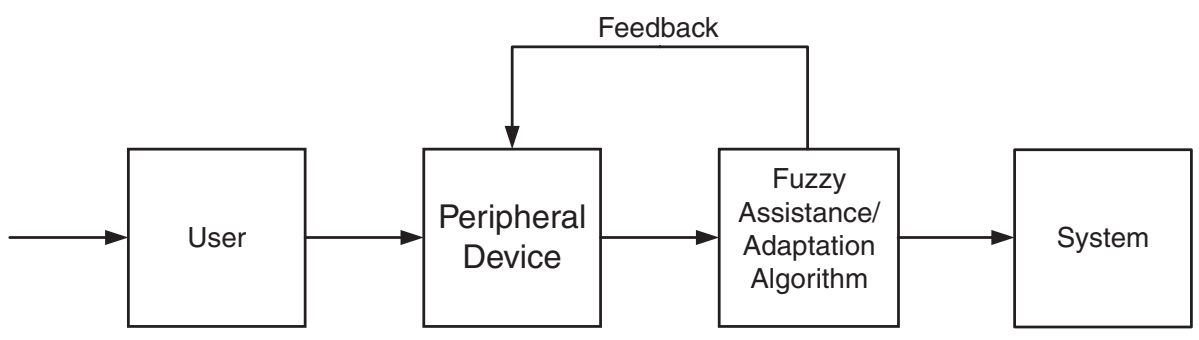

Fig. 14. Fuzzy assistance/adaptation model.

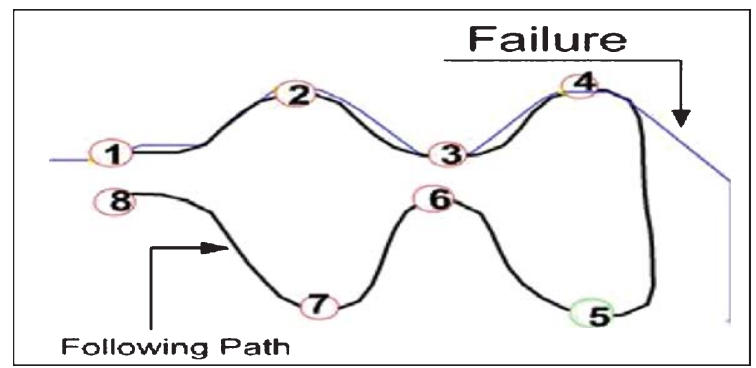

Fig. 15. Trajectory of impaired patient.

\section{Assistance and adaptation services}

PLEIA assistance and adaptation services defined in Section 3 are implemented using Fuzzy logic (Fig. 14).
PLEIA defines multiple assistance modes: the information feedback mode, the human supervision mode, the computer assistance mode, and the adaptation mode.

In the case of the Assistance Service, the user can get online force assistance from the joystick to reach the target. This force can be attraction force toward a target or path, or repulsion force to avoid an obstacle. The assistance service implementation is based on fuzzy logic which is applied for both $x$ (horizontal) and $y$ (vertical) axes, with some difference in fuzzy rules for each axis. Four input variables and one output variable are defined for each axis, and a fuzzy set with different values is defined for each input or output variable. The defined fuzzy rules are based on a single or on a combination of the input variables. They consist of specific fuzzy rules for each of the $x$ or $y$ axis, and of common
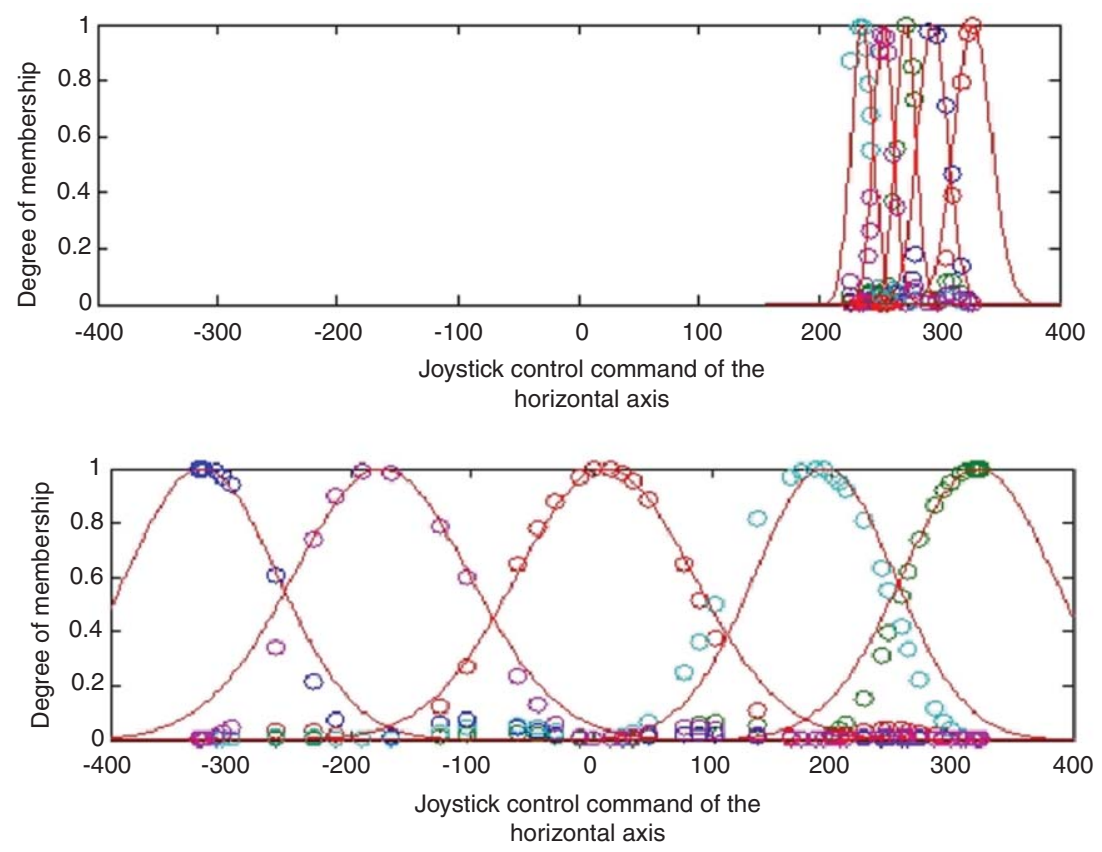

Fig. 16. Fuzzy sets before and after the rehabilitation (horizontal axis). 
rules for both $x$ and $y$ axes. These rules were chosen to give the best possible assistance results.

The online Adaptation service is similar to the assistance service mentioned above, but in this case the fuzzy logic rules are changed online during the exercise. Online adaptation is implemented using the fuzzy reinforcement learning technique [26] as follows: At each time $t$, the system identifies its state $\mathrm{S}$ and generates an action $\mathrm{A}$ to be executed. The efficiency of this action is modeled by its reinforcement as a scalar index $\mathrm{K}_{i}$ (where $i$ is number of the fuzzy rule), and the Fuzzy Reinforcement Learning algorithm maximizes this reinforcement online. The major difference between the online adaptation service and the assistance service is in defining the reinforcement $\mathrm{K}_{i}$, which has a default value equal to 1 . If rule $i$ is doing right to reach the target, $\mathrm{K}_{i}$ is augmented by 0.1 (compensated), and if rule $\mathrm{i}$ is doing wrong, $\mathrm{K}_{i}$ is reduced by 0.1 (punished) and no compensation is done. In this way, the fuzzy rules are automatically adapted during the exercise.

Adaptation can also be done offline, in which case the fuzzy logic rules are changed after the exercise. This service is implemented using the Fuzzy C-mean algorithm of the Fuzzy Clustering technique [27], which is suitable for predefined problems.

For illustration purposes, this method is applied to a patient with no ability to move the joystick to the left. This patient is an eight years old girl suffering of quadriplegia. Figure 15 shows the impossibility of the user to follow the path after target 4 . In this case, 5 fuzzy sets are defined to describe a patient's movement in the horizontal direction. As a first step, the fuzzy module takes as input the joystick command of the user and calculates the membership degree $u[i]$ of this command for each set.

Figure 16 shows the clustering of the rehabilitated command. We notice that the command is distributed in the sensibility range of the joystick. In other words, the patient is now able to navigate on the screen in the fourth direction without any problem.

\section{Conclusion}

In this work, PLEIA evaluation and assistance multiplatform for people with disabilities is presented. PLEIA is currently operational and under evaluation by designers, therapists and patients. As a result of such evaluation, PLEIA is being improved and extended to include additional features. Currently, an investigation is being done using PLEIA on multiple normal and quadriplegic subjects in Hôpital National Saint Maurice, France. This study is concerned with usage of different computer pointing devices (mouse, joystick, and trackball). New quantitative indicators are being tested. Results of this investigation will be published soon.

\section{References}

[1] J. Winters, X. Feng, Y. Wang, L. Johnson and J. Foil, Progress toward universal interface technologies for telerehabilitation, Proceedings of the 26th Annual International Conference of the IEEE EMBS, San Francisco, CA, USA, pp. 4777-4780, 2004.

[2] X. Feng and J. Winters, UniTherapy: A computer-assisted motivating neurorehabilitation platform for teleassessment and remote therapy, Proceedings of the 2005 IEEE 9th International Conference on Rehabilitation Robotics, Chicago, IL, USA, pp. 349-352, 2005.

[3] I. Mougharbel, A. El Hajj, H. Artail and C. Riman, Remote lab experiments models: a comparative study, International Journal of Engineering Education 22(4) (2006), 849-857.

[4] A. El Hajj, I. Mougharbel and C. Riman, On the design and implementation of remote computer controlled laboratory experiments, CIBITIC Conference, Beirut, Lebanon, 2004.

[5] G. Burdea, Invited paper: Low-cost telerehabilitation, Proceedings of Third Int. Workshop on Virtual Rehabilitation, Switzerland, pp. 3297-3300, 2004.

[6] X. Feng and J. Winters, Progress towards a service-oriented universal access telerehabilitation platform, Proceedings of the 2005 IEEE 9th International Conference on Rehabilitation Robotics, Chicago, IL, USA, pp. 357-360, 2005.

[7] S. Keates, F. Hwang, et al., Cursor measures for motion-impaired computer users, Proceedings of the fifth International ACM Conference on Assistive Technologies 2002, Edinburgh, Scotland, 2002.

[8] D.J. Reinkensmeyer, C.T. Pang, J.A. Nessler and C.C. Painter, Web-based telerehabilitation for the upper extremity after stroke, IEEE Transactions on Neural Systems and Rehabilitation Engineering 10(2) (2002), 102-108.

[9] J.E. Deutsch, J.A. Lewis and G. Burdea, Technical and patient performance using a virtual reality-integrated telerehabilitation system: Preliminary finding, IEEE Transactions on Neural Systems and Rehabilitation Engineering 15(1) (2007), 30-35.

[10] D.P. Allen, J.R. Playfer, et al., On the use of low-cost computer peripherals for the assessment of motor dysfunction in Parkinson's disease-quantification of bradykinesia using target tracking tasks, IEEE Transactions on Neural Systems and Rehabilitation Engineering 15(2) (2007), 286-294.

[11] E.F. LoPresti and D.M. Brienza, Adaptive Software for head-operated computer controls, IEEE Transactions on Neural Systems and Rehabilitation Engineering 12(1) (2044), 102-111.

[12] B. Myers, J. Wobbrock, et al., Using handhelds to help people with motor impairments, Proceedings of the Fifth International ACM Conference on Assistive Technologies 2002, Edinburgh, Scotland, 2002.

[13] T. Felzer and B. Freisleben, The hands-free wheelchair control system, Proceedings of the Fifth International ACM Conference on Assistive Technologies 2002, Edinburgh, Scotland, 2002. 
[14] D. Vanhooydonck, E. Demeester, M. Nuttin and H. van Brussel, Shared control for intelligent wheelchairs: an implicit estimation of the user intention, Proceedings of the ACER '03 1st Iinternational Workshop on Advances in Services Robotics, Bardolino, Italy, 2003.

[15] R.A. Andersen, S. Musallam, J.W. Burdick and J.G. Cham, Cognitive based neural prosthetics, Proceedings of the 2005 IEEE International Conference on Robotics and Automation, Barcelona, Spain, 2005.

[16] C. Yu-Luen, Application of tilt sensors in human-computer mouse itnerface for people with disabilities, IEEE Transactions on Neural Systems and Rehabilitation Engineering 9(3) (2001), 289-294.

[17] R.C. Simpson and S.P. Levine, Voice control of a powered wheelchair, IEEE Transactions on Neural Systems and Rehabilitation Engineering 10(2) (2002), 122-125.

[18] G. Burdea, Haptic feedback interfaces for rehabilitation, Proceedings State of the Science Conference on Telerehabilitation and Applications of Virtual Reality, Washington, DC, pp. 51-56, 2001.

[19] D. Jaffe, Using Virtual Reality and Telerehabilitation to Improve Walking in Individuals Following Stroke, Proceedings State of the Science Conference on Telerehabilitation and Applications of Virtual Reality, Washington, DC, pp. 57-59, 2001.

[20] C. Jadhav and V. Krovi, A Low-Cost Framework for Individualized Interactive Telerehabilitation, Proceedings of the 26th Annual International Conference of the IEEE EMBS, San Francisco, CA, USA, pp. 3297-3300, 2004.
[21] G. Songfend, G.G. Grindle, et al., Development and qualitative assessment of the GAME/sup Cycle/exercise system, IEEE Transactions on Neural Systems and Rehabilitation Engineering 14(1) (2006), 83-90.

[22] U. Mali, N. Goljar and M. Munih, Application of haptic interface for finger exercise, IEEE Transactions on Neural Systems and Rehabilitation Engineering 14(3) (2006), 352-360.

[23] P. Hoppenot, E. Colle, O. Ait Aider and Y. Rybarczyk, A remote control manipulator assistance for disabled people, IFRATH 16-17, Paris, France, 2003.

[24] Y. Hirata, A. Hara and K. Kosuge, Motion Control of Passice-type Walking Support System based on Environment Information, Proceedings of the 2005 IEEE International Conference on Robotics and Automation, Barcelona, Spain, 2005.

[25] H. Peralta, C. Riman, et al., A Reconfigurable Evaluation and Assistance Platform for Handicapped, IROS 2006, Beijing, China, pp. 247-252, 2006.

[26] P. Y. Glorennec, Reinforcement learning: An overview, ESIT 2000, Aachen, Germany, 2000.

[27] M. Ramze, B. Llelieveldt and J. Reiber, A new cluster validity index for the fuzzy C-mean, Pattern Recognition Letters 19 (1998), 239-241.

[28] www.k-team.com

[29] X. Fend and J. Winters, A pilot study evaluating use of a computer-assisted neurohabilitatioin platform for upperextremity stroke assessment, Journal of NeuroEngineering and Rehabilitation 6 (2009), 15. 

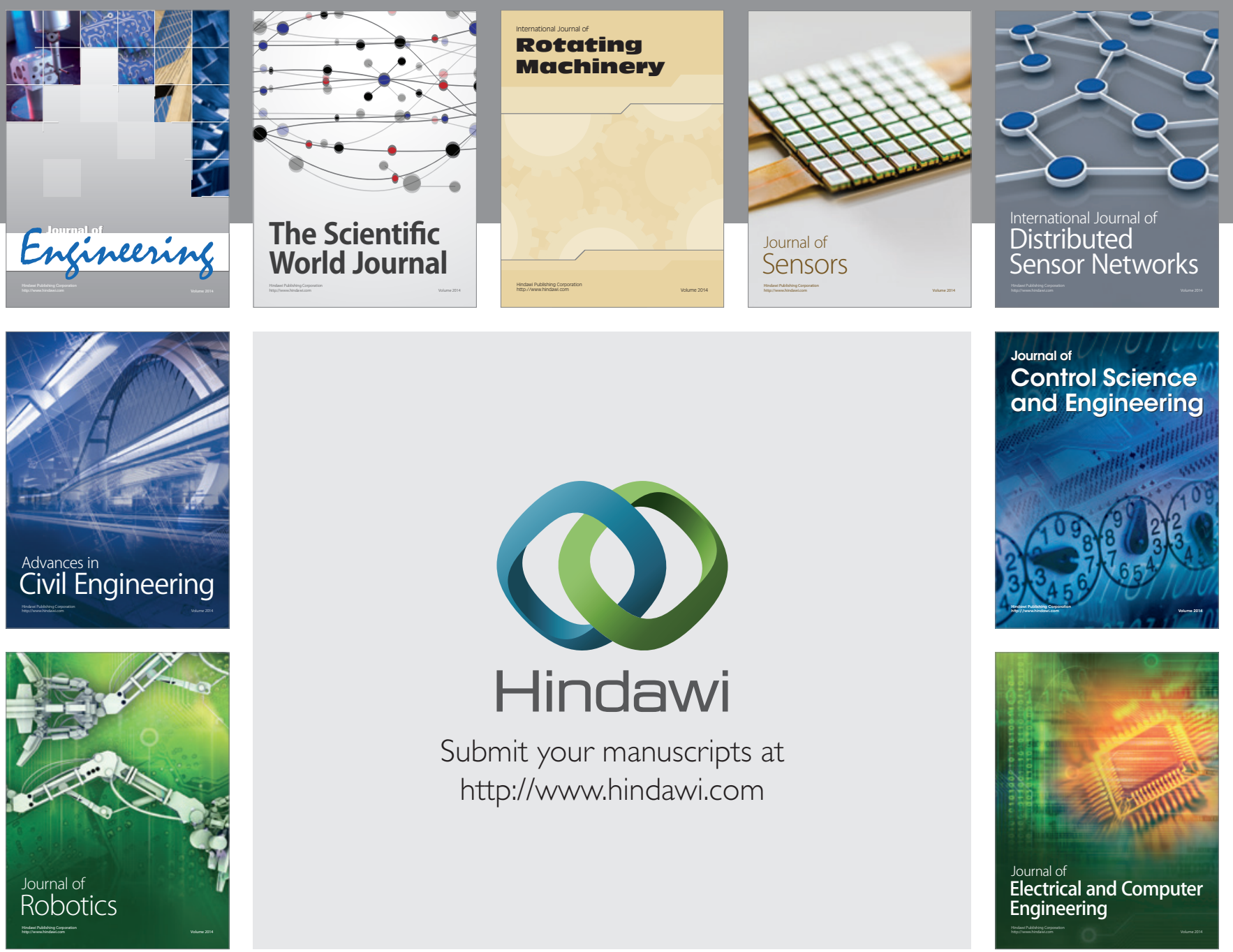

Submit your manuscripts at

http://www.hindawi.com
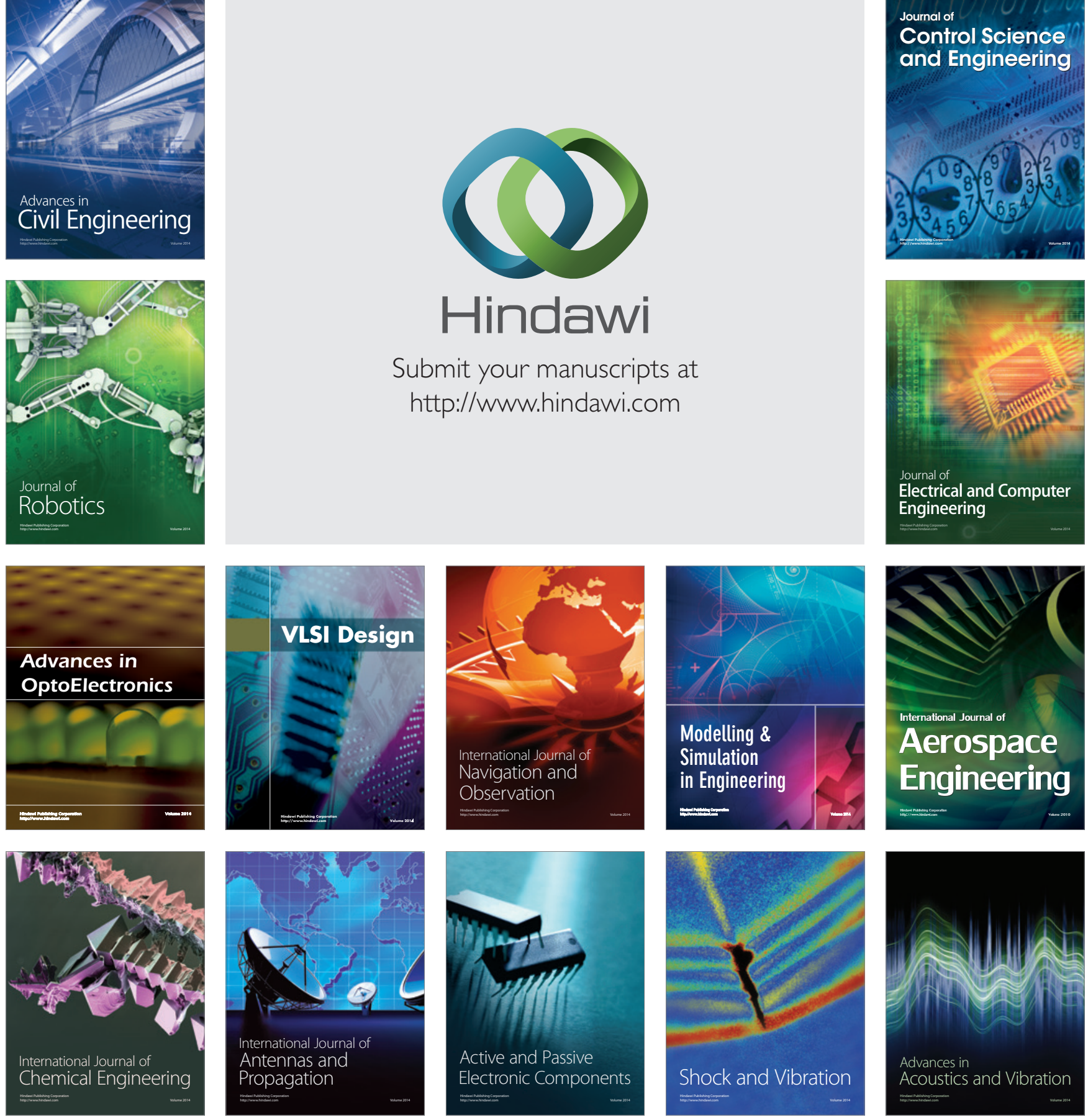